\section{O impacto da vacina pneumocócica PCV10 na redução da mortalidade por pneumonia em crianças menores de um ano em Santa Catarina, Brasil}

\author{
Impact of PCV10 pneumococcal vaccine on \\ mortality from pneumonia in children less than \\ one year of age in Santa Catarina State, Brazil \\ Impacto de la vacuna PCV10 neumocócica en la \\ reducción de la mortalidad por neumonía en niños \\ menores de un año en Santa Catarina, Brasil
}

\begin{abstract}
Resumo
O objetivo deste trabalho foi avaliar o impacto da vacina pneumocócica PCV10 na redução da mortalidade por pneumonia em crianças menores de um ano, em Santa Catarina, Brasil, considerando os quatro anos antes versus quatro anos após a introdução da vacina em 2010. Estudo com delineamento ecológico, realizado com dados do Sistema de Informações sobre Mortalidade e das coberturas vacinais em menores de um ano de idade. Os dados foram agrupados por município de residência e macrorregião. A taxa média de mortalidade por pneumonia em menores de um ano no período 2006-2009 versus 2010-2013 diminuiu de 29,69 para 23,40 por $100 \mathrm{mil}$, uma redução de $11 \%$. Porém, observa-se uma divisão entre regiões com redução (Grande Florianópolis, Sul, Planalto Norte e Nordeste) e outras com aumento do gradiente das taxas anuais (Oeste, Itajaí e Serra). No estado, verificou-se uma redução média da taxa de mortalidade, por pneumonia em menores de um ano, de 11\% após quatro anos da implantação da vacina como rotina no Programa Nacional de Imunizações, porém com efeitos heterógenos entre as regiões.
\end{abstract}

Vacinas Pneumocócicas; Pneumonia; Motalidade Infantil
Emil Kupek 1

Ilse Lisiane Viertel Vieira ${ }^{2}$

\author{
1 Departamento de Saúde \\ Pública, Universidade \\ Federal de Santa Catarina, \\ Florianópolis, Brasil. \\ 2 Departamento de Ciências \\ Biológicas e da Saúde e de \\ Ciências Sociais Aplicadas, \\ Universidade do Sul de Santa \\ Catarina, Tubarão, Brasil.
}

\author{
Correspondência \\ I. L. V. Vieira \\ Rua Prefeito Reinaldo Alves \\ 25, Palhoça, SC 88130-000, \\ Brasil. \\ iviertel@gmail.com
}




\section{Introdução}

Dados da Organização Mundial da Saúde (OMS) revelam que o número global de mortes entre crianças menores de cinco anos de idade foi de 6,9 milhões em 2011 1. O Brasil ocupou o 107o lugar no ranking mundial com 44 mil óbitos de crianças menores de cinco anos, ficando atrás de países como Argentina, Uruguai e Chile. É importante ressaltar que apesar desse elevado número de óbitos no Brasil, a taxa de mortalidade por mil nascidos em crianças menores de cinco anos passou de 58 para 16 durante o período de 1990-2011 1.

Com o decréscimo da mortalidade infantil, observou-se também um decréscimo significativo da mortalidade por pneumonia em crianças menores de cinco anos em todas as regiões do país. No período de 1991-2007, a redução média anual dos coeficientes de mortalidade por pneumonia na população menor de um ano e naquela entre 1 e 4 anos foi de 12 e 7 por 100 mil crianças, respectivamente. As regiões Sul e Sudeste apresentaram as maiores quedas, mas, apesar desta redução, a mortalidade por pneumonia foi responsável por cerca de $5 \%$ das mortes entre menores de 5 anos 2,3.

Esse declínio acelerado da mortalidade infantil pode estar associado à implementação, pelo Programa Nacional de Imunizações (PNI) 4,5, das vacinas tríplices viral (SCR), vacina oral de rotavírus humano (VORH), vacina meningocócica C (conjugada), vacina pneumocócica, dos Centros de Referência para Imunobiológicos Especiais e da ampliação do acesso aos serviços de saúde 6,7 .

No que se refere à vacina pneumocócica, esta foi introduzida no Brasil em 2002, com sete sorotipos (4, 6B, 9V, 14, 18C, 19F, 23F) para crianças a partir de dois meses, quando associada a situações predisponentes para contrair infecções pelo Streptococcus pneumoniae, denominada PCV7. Essa vacina se mostrou segura e imunogênica em diversos estudos, especialmente quando utilizada em esquema de quatro doses (2, 4, 6 meses de idade e booster entre os 12 a 15 meses) 6 . Estimase que o potencial de proteção para a doença pneumocócica invasiva causada pelos sorotipos constantes na vacina PCV7 seja de $70 \%$ e que apresente imunidade de grupo 7,8. A partir de 2008, 24 países incorporaram a vacina na rotina de imunização, principalmente na América do Norte e na Europa Ocidental 9,10. Em 2009 e 2010, a pneumocócica 13-valente (PCV13) foi licenciada no Chile, na Europa e nos Estados Unidos para uso em crianças com idades entre seis semanas e 71 meses, em substituição à PCV7 11,12,13.
A vacina pneumocócica 10-valente (PCV10), desenvolvida pela GlaxoSmithKline (GSK; Brentford, Reino Unido), obteve pré-qualificação da OMS em 200913 e foi incluída no calendário vacinal brasileiro para todas as crianças menores de dois anos em março de 2010 14,15. O Brasil foi o primeiro país a introduzir a PCV10 no PNI 14,15. A PCV10 é uma vacina conjugada de polissacarídeos pneumocócicos, que contém dez sorotipos de $S$. pneumoniae $(1,4,5,6 \mathrm{~B}, 7 \mathrm{~F}, 9 \mathrm{~V}, 14,18 \mathrm{C}, 19 \mathrm{~F}$ e 23F). O laboratório GSK disponibilizou a vacina para o Brasil até 2020 a baixo preço (US\$ 7,00/ dose), sendo que após o suprimento de $20 \%$ do quantitativo estabelecido, o valor diminuirá para metade. No mercado internacional, o preço gira em torno de US\$32,00/dose 16.

A inclusão da vacina PCV10 se configura como um grande avanço para a saúde pública brasileira, uma vez que protege as crianças contra doenças pneumocócicas invasivas (pneumonias bacterêmicas, meningite, sepses e artrite) e não invasivas (sinusite, otite média aguda, conjuntivite, bronquite e pneumonia) 14. A escolha dos sorotipos é o resultado de pesquisas desenvolvidas pelo Instituto Adolfo Lutz (São Paulo, Brasil), que compõe a rede de vigilância laboratorial do S. pneumoniae para os países da América Latina, e que no período de 2000-2008 analisou 4.376 cepas de pneumococo, sendo que $62 \%$ delas foram isoladas de pacientes com meningite, $28 \%$ de pacientes com pneumonia, $2 \%$ de pacientes com bacteremia e $8 \%$ com outras doenças causadas por S. pneumoniae 14 .

Com a introdução da vacina conjugada contra S. pneumoniae nos grupos prioritários de 0 a 2 anos, espera-se uma redução da morbimortalidade e também da colonização nasofaríngea, cuja taxa estimada foi de $40 \%$ em estudos no Brasil 9,17. Um estudo realizado no México de 20022004 avaliou a colonização nasofaríngea após a vacinação com PCV7, e mostrou uma rápida e adequada resposta imune que resultou na diminuição da presença dos sorotipos vacinais 18 . A proteção indireta da vacina reduz a morbimortalidade também em crianças não vacinadas e adultos 9

O objetivo do presente estudo foi o de avaliar, preliminarmente, o impacto da implantação da vacina PCV10 no Estado de Santa Catarina, Brasil, em março de 2010, na mortalidade por pneumonia em crianças menores de um ano, considerando os períodos de 2006-2009 versus 2010-2013. 


\section{Métodos}

Este foi um estudo com delineamento "ecológico", utilizando os dados anuais secundários por município para cálculo da cobertura vacinal e das taxas de mortalidade por pneumonia em menores de um ano no período de 2006-2013. Posteriormente, os dados foram agregados por macrorregiões do Estado de Santa Catarina. Identificou-se todos os óbitos por pneumonia em menores de um ano de idade, o número de nascidos vivos e as coberturas vacinais com a vacina PCV10 em menores de um ano.

As fontes de dados sobre nascidos vivos, óbitos e número de doses aplicadas das vacinas foram o Sistema de Informações sobre Nascidos Vivos (SINASC), o Sistema de Informações sobre Mortalidade (SIM) e o Sistema de Informação do Programa Nacional de Imunizações (SI-PNI) do Ministério da Saúde, disponibilizados pelo Departamento de Informática do Sistema Único de Saúde (DATASUS. http://www.datasus.gov.br, acessado em 21/Dez/2013).

Os óbitos foram classificados segundo os critérios de óbitos por pneumonia (dados agrupados J13, J15 e J18) da 10a revisão da Classificação Estatística Internacional de Doenças (CID-10).

A cobertura vacinal é definida pelo PNI como o percentual da população-alvo coberta com o esquema vacinal completo. A cobertura vacinal para a PCV10 em crianças menores de um ano de idade foi calculada utilizando-se como numerador o total de terceiras doses da vacina e como denominador o número de nascidos vivos. Embora para essa vacina exista o esquema com apenas duas doses, a depender da idade da criança ao iniciar o esquema vacinal, isto não foi avaliado nesta análise por representar uma fração muito pequena da população.

As variáveis independentes deste estudo foram a cobertura vacinal anual segundo município e imunobiológico (PCV10) no período 20102013 e a localização geográfica (sete macrorregiões do estado: Oeste, Itajaí, Planalto Norte, Nordeste, Planalto Serrano, Grande Florianópolis e Sul). O desfecho de interesse foi a taxa anual de mortalidade por pneumonia, conforme a CID10, em crianças menores de um ano. Com base nisso, calculou-se a taxa média por período anterior e posterior à vacina, dividindo-se o total dos óbitos pelo total dos expostos por período, assim como a tendência temporal das taxas anuais em cada período.

A estatística descritiva incluiu a representação tabular das taxas médias e a representação gráfica da tendência temporal das taxas de mortalidade por ano, nos períodos antes (2006-2009) e depois (2010-2013) da vacinação.
A razão das taxas médias de mortalidade por período foi calculada via regressão de Poisson para cada período separadamente, sempre ajustado por número de nascidos vivos. A diferença em tendência temporal entre os períodos antes e depois da vacinação com PCV10 foi calculada comparando-se os gradientes das taxas anuais para cada período. Os gradientes foram estimados com regressão polinomial 19, com termos de segundo e terceiro graus para linearizar as variações anuais bastante irregulares (bimodais na maioria das regiões). O valor inverso da variância das estimativas anuais representa a precisão das taxas anuais observadas, e foi utilizado como peso para determinar a tendência temporal destas em termos de um gradiente. Essa comparação dos gradientes linearizados, para detectar mudanças em tendência temporal da taxa de mortalidade por período, fez-se necessária porque a mesma média do período pode ser decorrente tanto de uma série das taxas anuais crescentes quanto decrescentes. Além do intervalo de 95\% de confiança (IC95\%) da diferença entre os gradientes por período, testou-se a hipótese da redução de mortalidade por pneumonia de no mínimo $20 \%$ no período após a vacinação. Esse critério foi fundamentado na eficácia da vacina em torno de $80 \%$ na diminuição da forma mais grave (invasiva) da pneumonia no Brasil 18. A significância estatística dessa hipótese foi calculada pelo teste t, isto é, dividindo-se a diferença entre os gradientes com o erro padrão da diferença e usando-se a curva normal para determinar as probabilidades de interesse. O software Stata, versão 12 (StataCorp LP, College Station, Estados Unidos), foi utilizado para os cálculos.

Como o presente trabalho foi realizado exclusivamente com dados agregados dos municípios, ele não se caracteriza como um estudo envolvendo seres humanos, sendo dispensada a apreciação por Comitê de Ética em Pesquisa.

\section{Resultados}

Em Santa Catarina, a vacina PCV10 foi implantada para todas as crianças menores de dois anos em março de 2010. Verificou-se um aumento gradativo das coberturas com essa vacina: em 2010 apenas 2,03\% dos municípios alcançaram $100 \%$ de cobertura, comparados com 53,59\% em 2013 (DATASUS. http://www.datasus.gov.br, acessado em 21/Dez/2013). A cobertura de PCV10 no estado aumentou de $62,15 \%$ em 2010 para $94,66 \%$ em 2011, 93,36\% em 2012 e 93,77\% em 2013. A cobertura média entre as regiões do estado variou pouco, entre $82,75 \%$ na Grande Florianópolis e $88,45 \%$ no Oeste do estado. 
Foram registrados 88 óbitos no período 2006 2009 e 82 no período 2010-2013. No estado, a taxa média de mortalidade por pneumonia em menores de um ano diminuiu de 29,69 para 23,40 por 100 mil no quadriênio em que ocorreu a vacinação, comparado com o quadriênio anterior, o que representa uma redução de $11 \%$ (Tabela 1). Embora a redução do número de óbitos fosse pequena, houve um aumento do número de nascidos vivos de $4,65 \%$, o que contribuiu para a redução da taxa média no último quadriênio. As regiões de Serra e do Sul, seguidas da Nordeste, apresentaram as maiores taxas de mortalidade por pneumonia entre 2006-2009, sendo as primeiras seguidas por uma considerável redução, enquanto a terceira continuou subindo no quadriênio posterior (Tabela 1, Figura 1).

A ocorrência de poucos óbitos por pneumonia ao longo dos anos, o fato de estarem distribuídos em apenas 36 dos 295 municípios do Estado de Santa Catarina e as características diferenciadas de cada região justificam a análise estratificada nas sete regiões. Observa-se também um grande número de municípios de pequeno porte, sem ocorrência de óbitos por pneumonia. Em contrapartida, dos municípios com mais de 50 mil habitantes apenas Concórdia não apresentou óbito por pneumonia no período analisado. Nos municípios de pequeno porte com até trinta nascidos vivos por ano, a taxa de mortalidade por pneumonia foi zerada após a vacinação com PCV10, enquanto naqueles com 51 a 100 nascidos vivos por ano a taxa foi reduzida para um terço do que era antes da vacinação. Um aumento da mortalidade foi observado apenas nos municípios com número de nascidos vivos entre 31 e 50 (Figura 2).

Embora a variação da taxa anual de mortalidade por pneumonia fosse bastante irregular (muitas vezes bimodal), os modelos da regressão polinomial conseguiram aproximar esta variação com uma boa precisão (Figura 1). Observouse uma queda na taxa anual de mortalidade por pneumonia por 100 mil até o ano de 2010, em 2011 ocorreu uma pequena elevação em cinco regiões do estado (Oeste, Itajaí, Grande Florianópolis, Nordeste e Sul) e posteriormente uma nova queda, exceto nas regiões Oeste, Serra e Nordeste.

As tendências para cada período foram comparadas por meio dos gradientes linearizados e seus intervalos de confiança (Tabela 2). Percebese uma clara divisão entre as regiões com uma redução significativa entre os quadriênios (Grande Florianópolis, Sul, Planalto Norte) e aqueles com aumento significativo do gradiente das taxas anuais (Oeste, Itajaí e Serra). Embora a redução do gradiente na região Nordeste não atingiu usual nível de 95\% de confiança para rejeitar a hipótese de nenhuma diferença entre os períodos comparados, a hipótese de uma redução de no mínimo $20 \%$ mostrou-se altamente plausível com probabilidade maior de $99 \%$ (Tabela 2). No estado inteiro, a tendência de queda do gradiente das taxas anuais de mortalidade por pneumonia foi mais acentuada após a implantação da vacina PCV10 na rotina das imunizações com relação ao quadriênio anterior.

Tabela 1

Mortalidade por pneumonia, segundo região, no Estado de Santa Catarina, Brasil, nos quadriênios antes e após a introdução da vacina PCV10 em 2010.

\begin{tabular}{|c|c|c|c|c|c|c|}
\hline \multirow[t]{2}{*}{ Regiões } & \multicolumn{2}{|c|}{ 2006-2009 } & \multicolumn{2}{|c|}{$2010-2013$} & \multirow[t]{2}{*}{ Diferença percentual das taxas (\%) } & \multirow{2}{*}{$\begin{array}{l}\text { Cobertura vacina } \\
\text { (PCV10) *** }\end{array}$} \\
\hline & Óbitos * & TMM ** & Óbitos * & TMM ** & & \\
\hline Oeste & 20 & 27,73 & 18 & 24,69 & $-11(-53,68)$ & 88,45 \\
\hline Itajaí & 17 & 21,86 & 18 & 21,18 & $-3(-50,88)$ & 86,46 \\
\hline Grande Florianópolis & 7 & 13,71 & 9 & 16,21 & $18(-56,22)$ & 82,75 \\
\hline Serra & 12 & 71,47 & 5 & 31,34 & $-56(-85,24)$ & 84,17 \\
\hline Nordeste & 14 & 28,79 & 19 & 36,01 & $25(-37,15)$ & 85,54 \\
\hline Sul & 14 & 30,41 & 9 & 18,88 & $-38(-73,43)$ & 85,42 \\
\hline Planalto Norte & 4 & 17,87 & 4 & 19,33 & $8(-73,33)$ & 86,95 \\
\hline Todas & 88 & 26,29 & 82 & 23,40 & $-11(-34,20)$ & 85,68 \\
\hline
\end{tabular}

TMM: taxa média de mortalidade.

* Óbitos por pneumonia;

** TMM por pneumonia por 100 mil habitantes no período;

*** Cobertura vacinal com a terceira dose da PCV10 em menores de 1 ano de idade no período 2010-2013. 
Tendência temporal da taxa de mortalidade (por 100 mil habitantes) por pneumonia em crianças menores de um ano de idade, segundo região, no Estado de Santa Catarina, Brasil, 2006-2013.

1a) Oeste

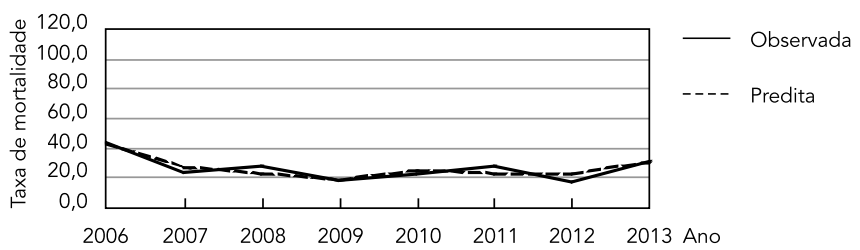

1c) Grande Florianópolis

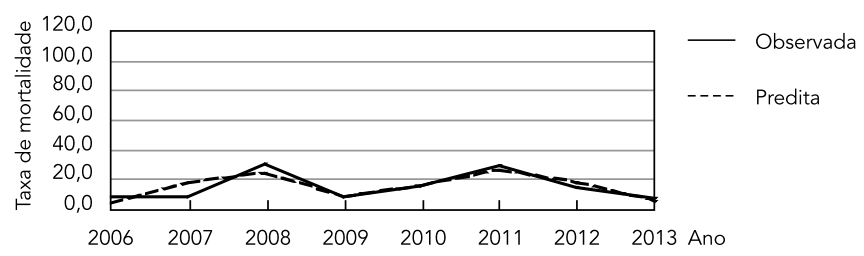

1e) Nordeste



1b) Itajaí

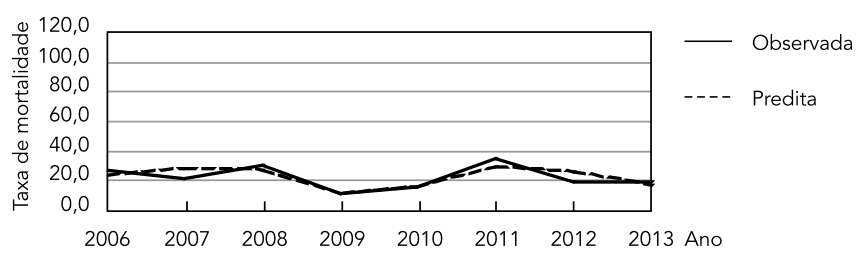

1d) Serra

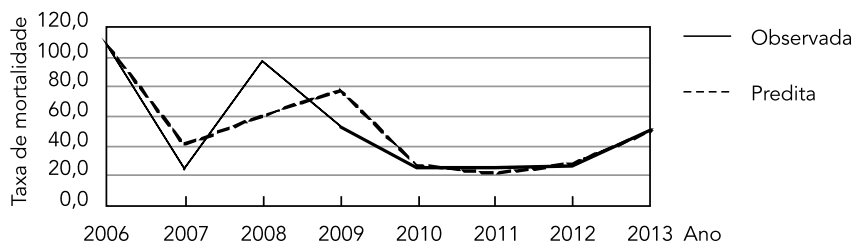

1f) Sul



1g) Planalto Norte

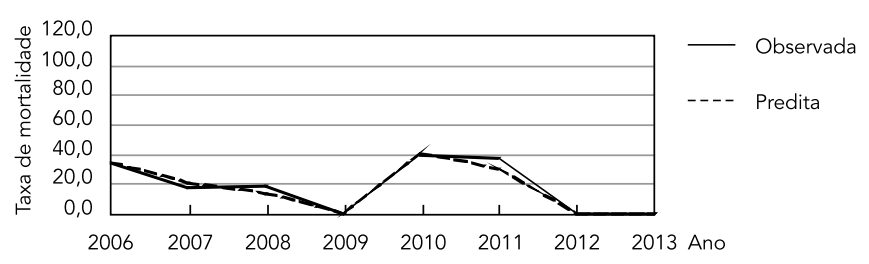

\section{Discussão}

Embora a redução da taxa média de mortalidade por pneumonia na comparação dos gradientes antes e depois da implantação da vacina
PCV10 não fosse estatisticamente significativa no estado como um todo, a análise estratificada mostra que a redução foi significativa nas regiões da Grande Florianópolis, Nordeste, Sul e Planalto Norte, e as regiões de Oeste, Itajaí e Serra 
Figura 2

Taxa de mortalidade por pneumonia por 100 mil menores de um ano segundo o número de nascidos vivos nos Municípios de Santa Catarina, Brasil (períodos 2006-2009 versus 2010-2013).

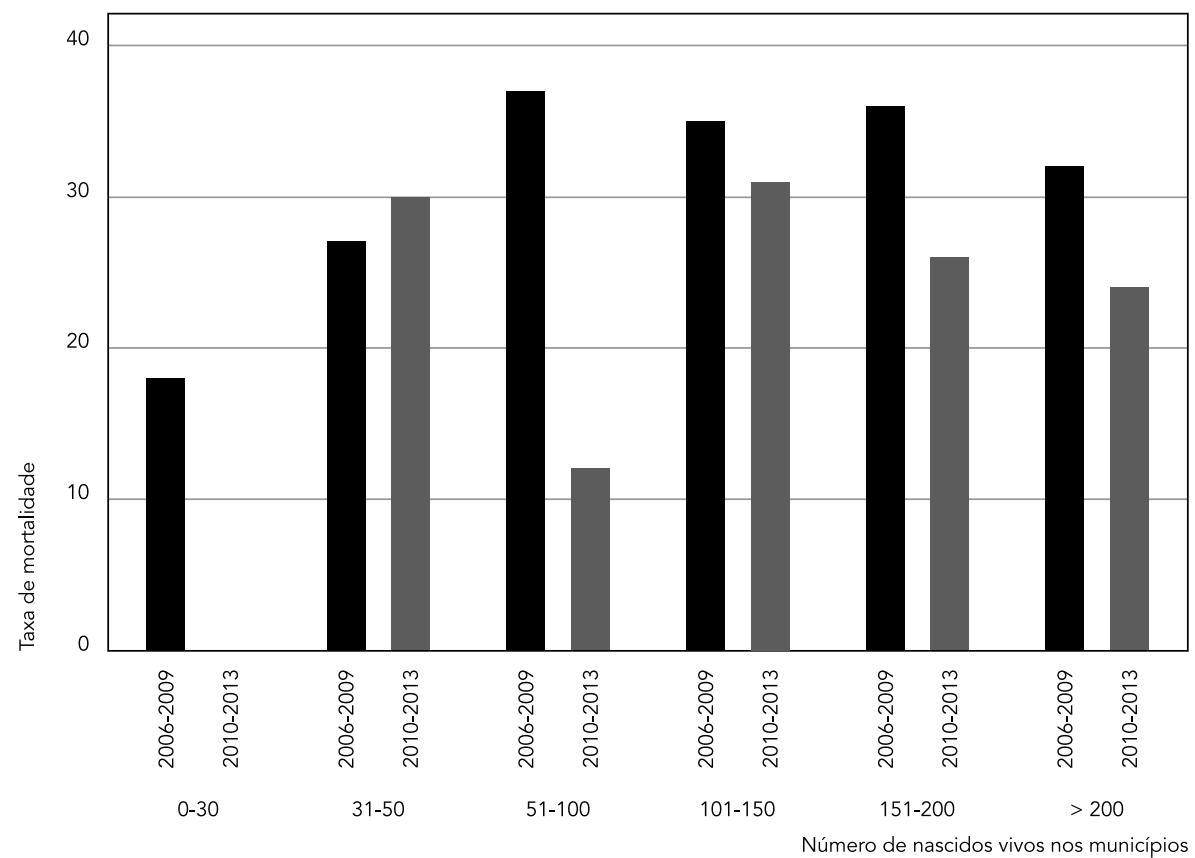

Tabela 2

Tendência temporal das taxas anuais de mortalidade por pneumonia, segundo região, no Estado de Santa Catarina, Brasil, antes e depois da vacinação com PCV10.

\begin{tabular}{|c|c|c|c|c|c|c|}
\hline \multirow[t]{2}{*}{ Regiões } & \multicolumn{3}{|c|}{ Tendência temporal (gradiente) por período * } & \multicolumn{2}{|c|}{ IC95\% da diferença } & \multirow{2}{*}{$\begin{array}{l}\text { Probabilidade de redução ** } \\
\qquad(20 \%)\end{array}$} \\
\hline & 2006-2009 & $2010-2013$ & Diferença & Mínimo & Máximo & \\
\hline Oeste & $-6,54$ & 0,98 & 7,52 & 2,09 & 12,95 & $<0,001$ \\
\hline Itajaí & $-3,34$ & $-1,23$ & 2,11 & $-3,75$ & 7,86 & 8,85 \\
\hline Grande Florianópolis & 3,20 & $-4,48$ & $-7,68$ & $-14,11$ & $-1,24$ & 99,91 \\
\hline Serra & $-6,58$ & 7,24 & 13,82 & 3,67 & 23,97 & $<0,001$ \\
\hline Nordeste & 15,45 & 10,80 & $-4,65$ & $-11,07$ & 1,78 & 99,99 \\
\hline Sul & 1,33 & $-9,79$ & $-11,12$ & $-17,47$ & $-4,76$ & 99,99 \\
\hline Planalto Norte & $-9,25$ & $-16,88$ & $-7,63$ & $-13,66$ & -1.60 & 99,97 \\
\hline Todas & $-0,39$ & $-1,24$ & $-0,85$ & $-6,09$ & 4.39 & 55,57 \\
\hline
\end{tabular}

IC95\%: intervalo de 95\% de confiança.

* Gradientes linearizados com regressão polinomial;

** Probabilidade de redução do gradiente da tendência temporal de no mínimo $20 \%$ no período após a implantação da vacina PCV10. 
seguiram a tendência oposta. Logo, o impacto da vacinação foi heterógeno, portanto a média do estado não é suficiente para elucidar a evolução da mortalidade no período analisado. As quatro regiões com a redução significativa reverteram a tendência de aumento da taxa anual no quadriênio anterior à vacinação para uma tendência de queda no quadriênio seguinte. Por exemplo, a tendência de aumento da mortalidade na Grande Florianópolis de 3,20 por 100 mil por ano foi revertida em queda de 4,48 por 100 mil por ano no período de vacinação com PCV10. No Planalto Norte, a tendência de diminuição da taxa de 9,25 no período anterior à vacinação foi acentuada para 16,88 por 100 mil por ano no período posterior. No estado como um todo, a redução anual da taxa de mortalidade de 0,39 no quadriênio sem vacinação acelerou para 1,24 por 100 mil no quadriênio com vacinação, embora o IC95\% desta redução seja compatível com uma variação não significativa entre os quadriênios. A magnitude da redução da tendência em taxa anual de mortalidade de $20 \%$, condizente com o efeito esperado da vacina, foi altamente provável para as regiões da Grande Florianópolis, Nordeste, Sul e Planalto Norte. Por outro lado, a tendência de aumento dessa taxa nas regiões Oeste, Itajaí e Serra é altamente preocupante e precisa ser revertida urgentemente.

A heterogeneidade do impacto da vacina também foi observada com relação ao número de nascidos vivos. A taxa de mortalidade por pneumonia foi reduzida consideravelmente após a vacinação com PCV10 nos municípios de pequeno porte com até trinta nascidos vivos por ano, enquanto naqueles com 51 a 100 nascidos vivos por ano a redução foi para um terço do que era antes da vacinação. Por outro lado, o benefício da vacina foi muito mais modesto nos municípios maiores com mais de cem nascidos vivos por ano, e ocorreu um aumento da mortalidade nos municípios com número de nascidos vivos entre 31 e 50. Esse resultado é sugestivo de um maior acompanhamento das condições de saúde das crianças em alguns municípios e possível dificuldade de acesso à vacina e aos serviços médicos em geral em outros, o que remete à necessidade de uma investigação mais específica.

Embora a vacina PCV10 tenha mostrado a eficácia de reduzir a incidência da pneumonia mais grave (invasiva) no Brasil de $83,9 \%$ causada por sorotipos vacinais 20 , seu efeito na redução da mortalidade depende muito da cobertura vacinal e da susceptibilidade/vulnerabilidade dos quase $15 \%$ dos menores de um ano, não vacinados no estado. Com a cobertura média semelhante entre as regiões, ganha força a hipótese de que a susceptibilidade à forma mais grave de pneumonia e/ou a dificuldade de acesso ao serviço médico para diagnóstico e tratamento adequados possam ter contribuído para a tendência crescente da mortalidade nas regiões Oeste, Itajaí e Serra, mesmo no quadriênio em que a vacina foi implementada. Um estudo caso-controle ou a investigação dos óbitos por pneumonia ocorridos nessa época poderia esclarecer essa questão.

Uma redução heterogênea também foi encontrada no estudo que avaliou a taxa de hospitalização por pneumonia em crianças entre 2 e 24 meses de idade nas cinco capitais brasileiras logo após a introdução da vacina PCV10. O resultado apresenta um declínio na taxa de hospitalização por pneumonia entre janeiro de 2005 e agosto de 2011 de $28,7 \%$ em Belo Horizonte (Minas Gerais), 27,4\% em Recife (Pernambuco) e 23,3\% em Curitiba (Paraná), o que não foi evidenciado em São Paulo e Porto Alegre (Rio Grande do Sul), onde a redução não foi significativa. Essas diferenças foram atribuídas às coberturas vacinais encontradas 21.

Dados do PNI apontam que no Brasil há áreas onde as coberturas vacinais ultrapassam $100 \%$, mas também são observadas baixas coberturas em municípios de uma mesma região. Essas contradições têm suscitado no Ministério da Saúde a necessidade de se realizar inquéritos nacionais de cobertura vacinal 22. Em Santa Catarina, o inquérito para avaliação da PCV10 em 2013 identificou uma cobertura vacinal de $86,98 \%$ em menores de um ano, um pouco menor do que a cobertura de $93,77 \%$ registrada no DATASUS (Sistema de Informação do Programa Nacional de Imunizações 2013. http://pni.datasus.gov.br/ consulta_mrc_13_selecao.php?sel=C02\&uf=SC, acessado em 21/Jul/2014) para esse ano.

Uma revisão realizada com base em 37 artigos publicados na Austrália, Europa e América do Norte sobre o impacto da PCV7 na redução da incidência de doenças pneumocócicas invasivas, também evidenciou diferenças importantes entre os países, sendo a menor redução registrada na Espanha de 39,9\%, e a maior de 99,1\% nos Estados Unidos 23. Em Gâmbia, um estudo randomizado com grupo controle acompanhou 22 mil crianças e evidenciou uma proteção conferida pela vacina PCV-9 contra pneumonia com confirmação radiológica de $27 \%$ em comparação com o grupo controle, reduzindo em $16 \%$ as mortes entre as crianças vacinadas 24 . No Uruguai, as taxas de hospitalização por pneumonia em crianças, menores de 14 anos de idade, apresentaram um declínio de $20,4 \%$ para internações por pneumonia comprovada, sendo significativa entre crianças de 6 a 35 meses de idade ${ }^{25}$. No Brasil, houve a redução da incidência de meningites por pneumococo por 100 mil menores de 1 ano 
de idade de 7,15 em 2010 para 5,7 em 2011, ano seguinte à implantação da vacina 26 .

Embora o impacto da vacina pneumocócica venha se mostrando positivo no controle da pneumonia no Brasil e em outros países da América Latina como Argentina, Uruguai e Chile, os sorotipos para compor a vacina precisam ser constantemente avaliados 25,27,28. Apesar dessa avaliação não estar sendo realizada no Estado de Santa Cataria, um estudo realizado em Goiânia (Goiás), no início de 2011, descreve a redução na colonização pelos sorotipos vacinais de $44 \%$ nas crianças que receberam as três doses da vacina, o que não foi observado naquelas que receberam apenas uma dose ou não foram vacinadas 29 .

A efetividade global da vacina PCV7 em trabalhos publicados varia de $31 \%$ a $89 \%$, dependendo principalmente dos sorotipos de pneumococo predominantes em cada lugar, mas sua efetividade é baixa em lugares onde menos de $70 \%$ dos sorotipos circulantes estão presentes na vacina 30 . Um estudo realizado no Uruguai mostrou uma redução no número de óbitos por otite, bacteremias, pneumonias e meningites após a implementação da vacina PCV7 31. Além disso, a vacina foi altamente custo-efetiva. Uma revisão sistemática do período 1990-2006 estimou que a vacina poderia ter evitado um total de 678.161 casos de otite média aguda, 175.932 de pneumonia e 2.768 casos de septicemia e meningite pneumocócicas em crianças menores de 5 anos de idade na América Latina e no Caribe. Anualmente, poderiam ter sido evitadas 9.478 mortes com a vacina PCV7, que poderia salvar 0,9 vida por mil crianças vacinadas e evitar um caso de doença pneumocócica para cada 80 crianças vacinadas 32 .

No mundo, a pneumonia é a principal causa de morte entre as crianças menores de cinco anos e é a segunda causa de morte nos principais estados brasileiros 20,33. Na última década, mesmo antes da implantação da vacina pneumocócica, ocorreu um declínio na taxa de mortalidade por infecções respiratórias agudas em crianças 34,35. Ainda assim, o impacto que a pneumonia exerce sobre a morbidade e a mortalidade é muito grande. O Ministério da Saúde vem desenvolvendo um estudo caso-controle multicêntrico para auxiliar no monitoramento da colonização em orofaringe pelo S. pneumoniae, identificando assim as cepas mais frequentes em crianças nos estados do Amazonas, Ceará, Pernambuco, Bahia, Minas Gerais, São Paulo, Paraná, Rio Grande do Sul, Goiás e o Distrito Federal 20. É preciso ressaltar que no presente estudo não foi possível especificar os sorotipos que causaram a pneumonia, mas apenas a associação entre o uso da vacina e a redução da mortalidade em menores de um ano.
Novos estudos são necessários com base no acúmulo de dados por um período mais longo, para verificar a necessidade de adequar a composição da vacina segundo os sorotipos circulantes e o papel da bacteremia na mortalidade atribuída à pneumonia e a evolução da mortalidade com maior precisão. Os estudos caso-controle podem ser uma opção viável desde que controlem os fatores relacionados ao acesso ao tratamento médico adequado. Todavia, não se deve ignorar a importância do acompanhamento precoce da implantação de novas vacinas para verificar se o efeito observado é compatível com o esperado.

Entre outras limitações do presente trabalho, destaca-se o curto período pós-intervenção (após a implantação da vacina) disponível para análise, o que reduz o poder do teste estatístico na comparação entre os dois períodos. As análises de séries mais longas poderão controlar o efeito dos diferentes esquemas vacinais e avaliar o impacto da imunidade de grupo. É importante destacar que séries temporais de comparação antes e depois pressupõem homogeneidade entre os grupos sob comparação, principalmente com relação a não utilização da vacina antes de 2010. Embora as vacinas pneumocócica 23 valente e PCV7 fossem utilizadas pelo PNI somente em situações especiais em crianças com maior risco de adoecer e naquelas atendidas em clínicas privadas, o número de crianças vacinadas foi muito baixo do ponto de vista populacional. A vacina pneumocócica 23 valente só pode ser aplicada em crianças maiores de dois anos, e a PCV7 apresentou cobertura vacinal com a terceira dose em menores de um ano de 1,06\%, 1,71\%, $2,75 \%$ e $4,17 \%$ nos quatro anos que antecederam a introdução da PCV10 no estado.

Também é improvável que a diminuição da mortalidade aqui apresentada resulte apenas de uma melhora do nível socioeconômico da população e do acesso aos serviços de saúde. Destacase que essa avaliação não implica a negação dos determinantes sociais, culturais e econômicos da mortalidade infantil. A melhora de nível socioeconômico da população foi gradativa, relativamente pequena e estável no período analisado. Acrescenta-se que o tratamento efetivo da pneumonia não apresentou mudanças significativas 34 após a introdução da vacina PCV10 que explicariam a magnitude da redução de mortalidade por pneumonia encontrada no presente estudo.

É preciso destacar também que o registro de óbito por pneumonia pode ser influenciado por outras infecções de vias aéreas não prevenidas pela vacina PCV10 35, tais como a influenza A (H1N1) que pode originar um quadro de pneumonia 36,37 . Porém, esse cenário não foi 
observado, pois apenas no Planalto Norte ocorreu uma pequena elevação na taxa de mortalidade por pneumonia no período da epidemia da influenza A (H1N1), e em todo o estado, apenas um óbito em criança menor de um ano de idade foi atribuído à influenza, no ano de 2009.

A qualidade dos dados secundários num estudo com delineamento ecológico é mais uma limitação do presente trabalho, principalmente com relação a possíveis erros de diagnóstico que atribuem a causa de óbito às outras doenças respiratórias que não a pneumonia, resultando em diagnósticos falsos positivos/negativos. Essa seria uma interpretação alternativa para a observada heterogeneidade do efeito vacinal, visando à possibilidade de uma associação espúria entre a implementação do programa de vacinação e a variação da mortalidade por pneumonia.

Apesar das limitações, a utilização das fontes oficiais com controle de qualidade (DATASUS) remete a uma qualidade razoável das informações 38. O uso da regressão polinomial permitiu captar as variações irregulares das taxas anuais de mortalidade por pneumonia e incluí-las na estimativa da tendência temporal. Além da comparação das médias no período, a comparação das tendências temporais antes versus depois da implementação da vacina é uma informação complementar importante para avaliar o impacto da vacinação. Como o impacto é gradativo e tipicamente exponencial em função do tempo após a implementação, a regressão polinomial utilizada no presente estudo é a mais indicada porque permite analisar modelos mais complexos do que as regressões linear e de Poisson.

Em conclusão, neste estudo observou-se uma redução da taxa média de mortalidade por pneumonia em menores de um ano de $11 \%$ nos primeiros quatro anos de implantação da vacina PCV10 no Estado de Santa Catarina. Porém, esse resultado é limitado pela heterogeneidade do impacto vacinal: as regiões da Grande Florianópolis, Nordeste, Sul e Planalto Norte registraram uma redução significativa da tendência de mortalidade, enquanto a tendência oposta foi observada nas regiões de Serra, Oeste e Itajaí. Medidas urgentes são necessárias para expandir o efeito benéfico da vacina para todas as regiões do estado.

\section{Colaboradores}

E. Kupek participou da concepção do estudo, delineamento, análise e interpretação dos dados e redação do artigo. I. L. V. Vieira colaborou da concepção do estudo, delineamento, coleta de dados e redação do artigo.

\section{Agradecimentos}

À Luciana Amorim, enfermeira do Programa Nacional de Imunizações (PNI) e à Diretoria de Vigilância Epidemiológica da Secretaria de Estado da Saúde de Santa Catarina. 


\section{Referências}

1. The State of the World's Children. Basic indicators. http://www.unicef.org/rightsite/sowc/pdfs/sta tistics/SOWC_Spec_Ed_CRC_TABLE\%201.\%20BA SIC\%20INDICATORS_EN_111309.pdf (acessado em 21/Dez/2013)

2. Rodrigues FE, Tatto RB, Vauchinski L, Leães LM, Rodrigues MM, Rodrigues VB, et al. Mortalidade por pneumonia em crianças brasileiras até 4 anos de idade. J Pediatr (Rio J.) 2011; 87:111-4.

3. Axelsson I, Silfverdal SA. Mortalidade por pneumonia entre crianças brasileiras: uma história de sucesso. J Pediatr (Rio J.) 2011; 87:85-7.

4. Guimarães TMR, Alves JGB, Tavares MMF. Impacto das ações de imunização pelo Programa Saúde da Família na mortalidade infantil por doenças evitáveis em Olinda, Pernambuco, Brasil. Cad Saúde Pública 2009; 25:868-76.

5. França ISX, Simplício DN, Alves FP, Brito VRS. Cobertura vacinal e mortalidade infantil em Campina Grande, PB, Brasil. Rev Bras Enferm 2009; 62:258-71.

6. Farhat CK, Cintra OAL, Tregnaghi MW. Vaccination and the respiratory tract: what should we know? J Pediatr (Rio J.) 2002; 78:195-204.

7. Maranhão AGK, Vasconcelos AMN, Trindade CM, Victora CG, Rabello Neto DL, Porto D, et al. Mortalidade infantil no Brasil: tendências, componentes e causas de morte no período de 2000 a 2010. In: Departamento de Análise de Situação de Saúde, Secretaria de Vigilância em Saúde, Ministério da Saúde, organizador. Saúde Brasil 2011: uma análise da situação de saúde e a vigilância da saúde da mulher. v. 1. Brasília: Ministério da Saúde; 2012. p. 163-82.

8. Pilishvili T, Lexau C, Farley MM, Hadler J, Harrison LH, Bennett NM, et al. Sustained reductions in invasive pneumococcal disease in the era of conjugate vaccine. J Infect Dis 2010; 201:32-41.

9. pneumoADIP; Pan American Health Organization; Sabin Vaccine Institute; Centers for Disease Control and Prevention. Ata do Simpósio Nacional de Vigilância: pneumococo e influenza. http://www. sabin.org/sites/sabin.org/files/Ata\%20do\%20Sim posio\%20Nacional\%20de\%20Vigilancia\%20_\%20 Pneumococo\%20e\%20Influenza.pdf (acessado em 19/Nov/2014).

10. Feijó RB, Cunha J, Krebs LS. Calendário vacinal na infância e adolescência: avaliando diferentes propostas. J Pediatr (Rio J.) 2006; 82:4-14.

11. DeStefano F, Pfeifer D, Nohynek H. Perfil de segurança das vacinas conjugadas pneumocócicas: revisão sistemática de pré e pós-licenciamento de dados. Rev Saúde Pública 2008; 86:373-80.

12. Centers for Disease Control and Prevention. Vaccines and preventable diseases: PCV13 (pneumococcal conjugate) vaccine recommendations, scenarios and Q\&As for health care professionals about PCV13 for immunocompromised adults. http://www.cdc.gov/mmwr/preview/mmwrhtml/ mm5909a2.htm (acessado em 03/Jan/2014).
13. Centers for Disease Control and Prevention. Invasive pneumococcal disease in young children before licensure of 13-valent pneumococcal conjugate vaccine - United States, 2007. MMWR Morb Mortal Wkly Rep 2010; 59:253-7.

14. Departamento de Vigilância Epidemiológica, Secretaria de Vigilância em Saúde, Ministério da Saúde. Informe técnico da vacina pneumocócica 10-valente (conjugada). Brasília: Ministério da Saúde; 2010. (Série A. Normas e Manuais Técnicos).

15. Ministério da Saúde. Programa Nacional de Imunizações. Brasília: Ministério da Saúde; 2011.

16. Homma A, Dagna CO. Economic impact of pneumococcal conjugate vaccination in Brazil, Chile, and Uruguay. Rev Panam Salud Pública 2008; 24:101-12.

17. Ferreira LLM, Carvalho ES, Berezin EN, Brandileone MC. Colonização e resistência antimicrobiana de Streptococcus pneumoniae isolado em nasofaringe de crianças com rinofaringite aguda. J Pediatr (Rio J.) 2001; 77:227-34.

18. Espinosa-de los Monteros LE, Aguilar-Ituarte F, Jiménez-Juárez RN, Rodríguez-Suárez RS, GómezBarreto D. Reemplazo de serotipos de Streptococcus pneumoniae en niños con vacuna conjugada antineumocóccica 7V en México. Salud Pública Méx 2010; 52:4-13.

19. Yang H, Novick SJ, LeBlond D. Testing assay linearity over a pre-specified range. J Biopharm Stat 2015; 25:339-50.

20. Domingues CMAS, Verani JR, Montenegro Renoiner EI, de Cunto Brandileone MC, Flannery B, Oliveira LH. Effectiveness of ten-valent pneumococcal conjugate vaccine against invasive pneumococcal disease in Brazil: a matched case-control study. Lancet Respir Med 2014; 2:464-71.

21. Afonso ET, Minamisava R, Bierrenbach AL, Escalante JJC, Alencar AP, Domingues CM, et al. Effect of 10-valent pneumococcal vaccine on pneumonia among children, Brazil. Emerg Infect Dis 2013; 19:589-97.

22. Mota E. Inquérito domiciliar de cobertura vacinal: a perspectiva do estudo das desigualdades sociais no acesso à imunização básica infantil. Rev Bras Epidemiol 2008; 11:125-8.

23. Myint TTH, Madhava H, Balmer P, Christopoulou $\mathrm{D}$, Attal S, Menegas D, et al. The impact of 7-valent pneumococcal conjugate vaccine on invasive pneumococcal disease: a literature review. Adv Ther 2013; 30:127-51.

24. Cutts FT, Zaman SM, Enwere G, Jaffar S, Levine OS, Okoko JB, et al. Efficacy of nine-valent pneumococcal conjugate vaccine against pneumonia and invasive pneumococcal disease in the Gambia: randomized, double-blind, placebo-controlled trial. Lancet 2005; 365:1139-46.

25. Hortal M, Estevan M, Meny M, Iraola I, Laurani H. Impact of pneumococcal conjugate vaccines on the incidence of pneumonia in hospitalized children after five years of its introduction in Uruguay. PLoS One 2014; 9:e98567. 
26. Domingues CMAS, Teixeira AMS. Coberturas vacinais e doenças imunopreveníveis no Brasil no período 1982-2012: avanços e desafios do Programa Nacional de Imunizações. Epidemiol Serv Saúde 2013; 22:9-27.

27. Gaiano A, Biscayart C, Vizzotti C. Introducción de la vacuna conjugada antineumocócica en el calendario nacional de vacunación en Argentina. Rev Argent Salud Pública 2012; 3:41-3.

28. López EL. Proyecciones de las vacunas anti neumocóccicas conjugadas en Latinoamérica. Rev Chil Infect 2001; 18:25-30.

29. Andrade AL, Ternes YM, Vieira MA, Moreira WG, Lamaro-Cardoso J, Kipnis A, et al. Direct effect of 10 -valent conjugate pneumococcal vaccination on pneumococcal carriage in children Brazil. PLoS One 2014; 9:e98128.

30. Guevara M, Barricarte A, Pérez B, Arriazu M, García Cenoz M, Castilla J. La vacuna neumocócica conjugada heptavalente (Prevenar). Diferencias en su efectividad en distintas poblaciones. An Sist Sanit Navar 2008; 31:171-92.

31. Giachetto Larraz G, Telechea Ortiz H, Speranza Mourine N, Giglio N, Cané A, Pírez García MC, et al. Costo-efectividad de la vacunación universal antineumocócica en Uruguay. Rev Panam Salud Pública 2010; 28:92-9.

\begin{abstract}
The aim of this study was to evaluate the impact of PCV10 pneumococcal vaccine on mortality from pneumonia in children less than one year of age in Santa Catarina State, Brazil, comparing the four years prior and the four years subsequent to the vaccine's introduction in 2010. This ecological study used data from the Mortality Information System and vaccination coverage of children less than one year. Data were grouped by municipalities of residence and regions. Average mortality from pneumonia in children under one year decreased from 29.69 to 23.40 per 100,000, comparing 2006-2009 and 2010-2013, or a reduction of 11\%. However there were differences between regions with a drop in mortality (Grande Florianópolis, Sul, Planalto Norte, and Nordeste) and others with an increase in the annual rates (Oeste, Itajai, and Serra). In short, the state as a whole showed $11 \%$ reduction in mortality from pneumonia in children less than one year of age, four years after implementing routine PCV10 vaccination in the National Immunization Program, but with heterogeneous effects when comparing regions of the state.
\end{abstract}

Pneumococcal Vaccines; Pneumonia; Infant Mortality
32. Valenzuela MT, O’Loughlin R, De La Hoz F, Gomez E, Constenla D, Sinha A, et al. A carga da doença pneumocócica entre crianças latino-americana e caribenha: revisão das evidências. Rev Panam Salud Pública 2009; 25:270-9.

33. Ahmad OB, Lopez AD, Inoue M. The decline in child mortality: a reappraisal theme papers. Bull World Health Organ 2000; 78:1175-91.

34. Berezin EN. Vacinas pneumocócicas e pneumonias. J Pediatr (Rio J.) 2012; 88:97-8.

35. Cillóniz C, Torre A. Entendimento da mortalidade em pneumonia pneumocócica bacterêmica. J Bras Pneumol 2012; 38:417-8.

36. Schuelter-Trevisol F, Dutra MC, Uliano EJM, Zandomênico J, Trevisol DJ. Perfil epidemiológico dos casos de gripe A na região sul de Santa Catarina, Brasil, na epidemia de 2009. Rev Panam Salud Pública 2012; 32:82-6.

37. Magalhães J, Pinho L, Mendes C, Dias A, Zilhão C, Garrido C, et al. Infecção por H1N1 num serviço de pediatria. Nascer Crescer 2012; 21:9-12.

38. Szwarcwald CL, Leal MC, Andrade CLT, Souza Jr. PRB. Estimação da mortalidade infantil no Brasil: o que dizem as informações sobre óbitos e nascimentos do Ministério da Saúde? Cad Saúde Pública $2002 ; 18: 1725-36$.

\section{Resumen}

El objetivo de este estudio fue evaluar el impacto de la PCV10 en la reducción de mortalidad por neumonía en niños menores de un año en Santa Catarina, Brasil, teniendo en cuenta los cuatro años anteriores, frente a los cuatro años posteriores a la introducción de la vacuna en 2010. Este estudio ecológico se hizo con datos del Sistema de Informaciones sobre Mortalidad y cobertura de vacunación en niños menores de un año de edad. Los datos se agruparon según el lugar de residencia y región. La tasa media de mortalidad por neumonía en niños menores de un año en el período 2006-2009, en comparación con 2010-2013, disminuyó de 29,69 a 23,40 por 100.000 habitantes, una reducción del $11 \%$. Sin embargo, hay una división entre las regiones con la reducción (Florianópolis, Sur, Norte y Nordeste Meseta) y otra con el aumento del gradiente de tasas anuales (West, Itajaí y Serra). En el estado hubo una reducción media de mortalidad por neumonía en niños menores de un año de un $11 \%$, tras cuatro años de aplicación de la vacuna en el Programa Nacional de Inmunización, pero con efectos heterogéneos entre regiones.

Vacunas Neumocócicas; Neumonía; Mortalidad Infantil
Recebido em 29/Ago/2014

Versão final reapresentada em 14/Abr/2015

Aprovado em 23/Jul/2015 